\title{
Decentralized rationing problems and the proportional rule
}

\author{
Josep M. Izquierdo*and Pere Timoner ${ }^{\dagger}$ \\ Department of Mathematical Economics, Finance and Actuarial Sciences, \\ University of Barcelona and Barcelona Economic Analysis Team, \\ Av. Diagonal, 690, 08034 Barcelona, Spain.
}

September 21, 2018

\begin{abstract}
Decentralized rationing problems are those in which the resource is not directly assigned to agents, but first allocated to groups of agents and then divided among their members. Within this framework, we define the decentralized proportional rule. We characterize it as the only solution that satisfies equal treatment of equals, provided claims are rational numbers. Finally, we prove that the proportional rule is the only solution that assigns the same allocation regardless of whether the resource is distributed directly to agents or in a decentralized manner (with agents grouped).
\end{abstract}

Keywords: rationing, claims problem, proportional rule, a priori unions, multi-issue allocation, non manipulability

JEL: D63.

${ }^{*}$ Corresponding author. E-mail: jizquierdoa@ub.edu

${ }_{\dagger}$ E-mail: ptimoner@ub.edu 


\section{Introduction}

A standard rationing situation ${ }^{1}$ is an allocation problem in which an amount of a scarce resource is directly allocated to claimants. In this paper we consider rationing problems when the resource is assigned indirectly. We suppose that agents are distributed in groups and we allocate the resource by using a two-stage procedure that involves, first, distributing the resource among the groups and, second, dividing it among their members. We call these situations decentralized rationing problems. The agents may be grouped for many reasons, including lack of information, geographical proximity, logistics, different typologies of agents, etc.

The model we analyse fits into the model of bankruptcy with a priori unions (Casas-Méndez et al., 2003). A related model to the one proposed in this paper is the multi-issue allocation (MIA) model, where each agent makes several claims related to different issues (e.g., Bergantiños et al., 2010, 2011; Lorenzo-Freire et al., 2010). In these papers, the resource is distributed between issues and, second, it is divided among agents. In fact, a decentralized rationing problem can be reinterpreted as a $M I A$ situation, where the groups play the role of issues and each agent (being a member of exactly one group) claims for only one issue.

In this decentralized context, it is relevant to understand which properties of rules are preserved from the centralized model and which ones are broken. In particular we will focus on the property of equal treatment of equals, which states that if two agents have equal claims (regardless of their group membership), then they should receive equal amounts. We show that there is only one rule that satisfies this property (provided claims are rational numbers): it is the decentralized (two-stage extension) proportional rule (see Theorem 1). Let us mention that, within the $M I A$ framework, Bergantiños et al. (2010) already provide a characterization of a two-stage extension of the proportional rule, but using the properties of non-advantageous transfer across issues and non-advantageous transfer within issues.

Another interesting feature to analyze is whether the allocation proposed by a rule is the same regardless the resource is distributed directly to agents or in a decentralized manner. We name these solutions decentralized consistent rules. In Theorem 2, we prove that decentralized consistency, without the anonimity requirement used in Moreno-Ternero (2009), is enough to characterize the proportional rule. Corollary 1 points out that decentralized consistency is equivalent to other concepts studied in the literature, such as strategy-proofness (O'Neill, 1982) and non manipulability² (de Frutos, 1999).

\section{Notations and definitions}

Let $\mathbb{N}$ be the set of natural numbers that we identify with the universe of potential agents, and let $\mathcal{N}$ be the family of all finite subsets of $\mathbb{N}$. We denote by $t$ the cardinality of a coalition $T \in \mathcal{N}$. Given a finite subset of agents $N=\{1,2, \ldots, n\} \in \mathcal{N}$, a standard rationing problem $(r, c)$ for $N$ aims to distribute an amount $r \geq 0$ among these $n$ agents with claims $c=\left(c_{1}, c_{2}, \ldots, c_{n}\right) \in \mathbb{R}_{+}^{N}$.

\footnotetext{
${ }^{1}$ These situations are also known in the literature as problems of adjudicating conflicting claims (see, for instance, the survey undertaken by Thomson, 2015).

${ }^{2}$ Non manipulability and strategy-proofness are equivalent.
} 
It is assumed that $r \leq \sum_{i \in N} c_{i}$. Let us denote by $\mathcal{R}^{N}$ the set of all standard rationing problems with agent set $N$, and by $\mathcal{R}=\cup_{N \in \mathcal{N}} \mathcal{D}^{N}$ the family of all standard rationing problems.

A standard rationing rule $F$ associates to each problem $(r, c) \in \mathcal{R}^{N}$ a unique allocation $F(r, c)=x=\left(x_{1}, x_{2}, \ldots, x_{n}\right) \in \mathbb{R}^{N}$ such that $0 \leq x_{i} \leq c_{i}$, for all $i \in N$, and $\sum_{i \in N} x_{i}=r$, where $x_{i}$ represents the payoff to agent $i \in N$. For instance, the proportional rule $P$ allocates the resource proportionally to the claims, i.e. $P_{i}(r, c)=\frac{r}{\sum_{i \in N} c_{i}} \cdot c_{i}$ for all $i \in N$.

In a decentralized setting the agent set $N$ is partitioned in $g$ groups. A partition $\mathcal{P}$ of $N$ is formed by a collection of non-empty subsets of $N,\left\{N_{1}, N_{2}, \ldots, N_{g}\right\}$, such that $N=\cup_{j \in\{1,2, \ldots, g\}} N_{j}$ with $N_{j} \cap N_{j^{\prime}}=\emptyset$, for all $j \neq j^{\prime} \in\{1,2, \ldots, g\}$ and $N_{j} \neq \emptyset$, for all $j \in\{1,2, \ldots, g\}$. Let us denote by $G=\{1,2, \ldots, g\}$ the set of groups. Associated to each group $j \in G$ we have a vector of claims $c^{j}=\left(c_{i}\right)_{i \in N_{j}} \in \mathbb{R}_{+}^{N_{j}}$, where $c_{i}$ represents the claim of agent $i$ of group $j$. Moreover, we denote by $C^{j}=\sum_{i \in N_{j}} c_{i}$ the aggregate claim of agents of group $j \in G$ and by $C^{\mathcal{P}}=\left(C^{1}, C^{2}, \ldots, C^{g}\right)$ the vector of aggregate claims.

Definition 1 Let $N=\{1,2, \ldots, n\} \in \mathcal{N}$ be a finite set of agents. A decentralized rationing problem for $N$ is a triple $(r, c, \mathcal{P})$, where $r \in \mathbb{R}_{+}$is the amount of resource, $c=\left(c_{1}, c_{2}, \ldots, c_{n}\right) \in$ $\mathbb{R}_{+}^{N}$ is the claims vector and $\mathcal{P}=\left\{N_{j}\right\}_{j \in G}$ is a partition of the agent set $N$, such that

$$
r \leq \sum_{j \in G} \sum_{i \in N_{j}} c_{i}=\sum_{i \in N} c_{i} \text { (scarcity condition). }
$$

Let us denote by $\mathcal{D}^{N}$ the set of all decentralized rationing problems with agent set $N$, and by $\mathcal{D}=\cup_{N \in \mathcal{N}} \mathcal{D}^{N}$ the family of all decentralized rationing problems. As we have mentioned in the Introduction, we suppose that the resource is not directly assigned to agents. It is first allocated to groups according to a standard rationing rule $F$ and, in a second stage, each group divides the amount received among its members according to the same rule $F$.

Definition 2 Let $F$ be a standard rationing rule. The decentralized rationing rule relative to $F$ is a function $\mathcal{F}$ that associates to each problem $(r, c, \mathcal{P}) \in \mathcal{D}^{N}$ a unique allocation $\mathcal{F}(r, c, \mathcal{P}) \in \mathbb{R}_{+}^{N}$ as follows: for all $j \in G$ and all $i \in N_{j}$,

$$
\mathcal{F}_{i}(r, c, \mathcal{P}):=F_{i}\left(F_{j}\left(r, C^{\mathcal{P}}\right), c^{j}\right) .
$$

An example of such rules is the decentralized proportional rule $P^{d}$ where $F=P$.

\section{Decentralized rules: a characterization result}

In the previous section we have defined the concept of decentralized rationing rule. Next we consider whether a decentralized rule satisfies the equal treatment of equals property.

Definition 3 A decentralized rationing rule $\mathcal{F}$ satisfies equal treatment of equals if for all $N \in \mathcal{N}$ and all $(r, c, \mathcal{P}) \in \mathcal{D}^{N}$, it holds

$$
\text { if } c_{i}=c_{i^{\prime}} \text {, then } \mathcal{F}_{i}(r, c, \mathcal{P})=\mathcal{F}_{i^{\prime}}(r, c, \mathcal{P}) \text {. }
$$


In the theorem below we state that, for the case in which all claims are rational numbers ${ }^{3}$, the only rule that satisfies equal treatment of equals is the decentralized proportional rule. We denote by $\mathcal{D}_{\mathbb{Q}}^{N}:=\left\{(r, c, \mathcal{P}) \in \mathcal{D}^{N} \mid c_{i} \in \mathbb{Q}_{+}\right.$, for all $\left.i \in N\right\}$ the domain of all decentralized rationing problems with agent set $N$ and all claims being rational numbers. The family of all these problems is denoted by $\mathcal{D}_{\mathbb{Q}}:=\cup_{N \in \mathcal{N}} \mathcal{D}_{\mathbb{Q}}^{N}$.

Theorem 1 A decentralized rationing rule $\mathcal{F}$ on $\mathcal{D}_{\mathbb{Q}}$ satisfies equal treatment of equals if and only if it is the decentralized proportional rule.

Proof. The proof of the "if" part is straightforward. Next, we prove the "only if" part. Let us first prove the case where claims are natural numbers, i.e. $c_{i} \in \mathbb{N}$, for all $i \in N$. We denote by $\mathcal{D}_{\mathbb{N}}^{N}$ this subset of problems. Let $(r, c, \mathcal{P}) \in \mathcal{D}_{\mathbb{N}}^{N}$ and let $\mathcal{F}$ be a decentralized rationing rule that satisfies equal treatment of equals. We must prove that, for all $i \in N$,

$$
\mathcal{F}_{i}(r, c, \mathcal{P})=P_{i}^{d}(r, c, \mathcal{P})
$$

where $\mathcal{P}=\left\{N_{j}\right\}_{j \in G}$ is an arbitrary partition of $N$. To this end, consider the associated decentralized rationing problem $(r, \widehat{c}, \widehat{\mathcal{P}}) \in \mathcal{D}_{\widehat{N}}^{\widehat{N}}$ where

- $\widehat{N}=\{1,2, \ldots, \widehat{n}\}$ with $\widehat{n}=\sum_{i \in N} c_{i}$,

- $\widehat{c}_{k}=1$, for all $k \in \widehat{N}$ and

- $\widehat{\mathcal{P}}=\left\{\widehat{N}_{j}\right\}_{j \in G}$ is such that $\left|\widehat{N}_{j}\right|=\sum_{i \in N_{j}} c_{i}=C^{j}$, for all $j \in G$.

Notice that

$$
\widehat{C}^{\widehat{\mathcal{P}}}=\left(\widehat{C}^{1}, \widehat{C}^{2}, \ldots, \widehat{C}^{g}\right)=\left(C^{1}, C^{2}, \ldots, C^{g}\right)=C^{\mathcal{P}} .
$$

Since all claims are equal $\left(\widehat{c}_{k}=1\right.$, for all $\left.k \in \widehat{N}\right)$ and $\mathcal{F}$ satisfies equal treatment of equals it is straightforward that, for all $k \in \widehat{N}$,

$$
\mathcal{F}_{k}(r, \widehat{c}, \widehat{\mathcal{P}})=\frac{r}{\widehat{n}}=\frac{r}{\sum_{i \in N} c_{i}} .
$$

At this point, by (1) and (2), we have that, for all $j \in G$,

$$
F_{j}\left(r, C^{\mathcal{P}}\right)=F_{j}\left(r, \widehat{C}^{\widehat{\mathcal{P}}}\right)=\sum_{k \in \widehat{N}_{j}} \mathcal{F}_{k}(r, \widehat{c}, \widehat{\mathcal{P}})=\left|\widehat{N}_{j}\right| \cdot \frac{r}{\sum_{i \in N} c_{i}}=r \cdot \frac{C^{j}}{\sum_{i \in N} c_{i}}=P_{j}\left(r, C^{\mathcal{P}}\right) .
$$

Next, take an arbitrary $j \in G$ and denote $r_{j}=F_{j}\left(r, \widehat{C}^{\widehat{\mathcal{P}}}\right)$. Then, consider the standard rationing problem $\left(r_{j}, c^{j}\right) \in \mathcal{R}_{\mathbb{N}}^{N_{j}}$, and associate with it a decentralized rationing problem $\left(r_{j}, \widetilde{c}, \widetilde{\mathcal{P}}\right) \in$ $\mathcal{D}^{\widetilde{N}}$ where

- $\widetilde{N}=\widehat{N}_{j}$,

- $\widetilde{c}_{k}=1$, for all $k \in \tilde{N}$ and

- $\widetilde{\mathcal{P}}=\left\{\widetilde{N}_{i}\right\}_{i \in N_{j}}$ is such that $\left|\widetilde{N}_{i}\right|=c_{i}$, for all $i \in N_{j}$.

\footnotetext{
${ }^{3}$ We denote by $\mathbb{Q}$ the set of rational numbers.
} 
Notice that

$$
\widetilde{C}^{\widetilde{\mathcal{P}}}=\left(c_{i}\right)_{i \in N_{j}}=c^{j}
$$

Since $\mathcal{F}$ satisfies equal treatment of equals, we obtain that, for all $k \in \widetilde{N}$,

$$
\mathcal{F}_{k}\left(r_{j}, \widetilde{c}, \widetilde{\mathcal{P}}\right)=\frac{r_{j}}{|\widetilde{N}|}=\frac{r_{j}}{\left|\widehat{N}_{j}\right|}=\frac{r_{j}}{\sum_{i \in N_{j}} c_{i}}
$$

Thus, by (4) and (5), for all $i \in N_{j}$,

$$
\begin{aligned}
\mathcal{F}_{i}(r, c, \mathcal{P}) & =F_{i}\left(r_{j}, c^{j}\right)=F_{i}\left(r_{j}, \widetilde{C}^{\widetilde{\mathcal{P}}}\right)=\sum_{k \in \widetilde{N}_{i}} \mathcal{F}_{k}\left(r_{j}, \widetilde{c}, \widetilde{\mathcal{P}}\right) \\
& =\left|\widetilde{N}_{i}\right| \cdot \frac{r_{j}}{\sum_{i \in N_{j}} c_{i}}=r_{j} \cdot \frac{c_{i}}{\sum_{i \in N_{j}} c_{i}}=P_{i}\left(r_{j}, c^{j}\right) .
\end{aligned}
$$

Since $j \in G$ is an arbitrary group, by (3) and (6), it follows that, for all $j \in G$ and all $i \in N_{j}$,

$$
\mathcal{F}_{i}(r, c, \mathcal{P})=F_{i}\left(F_{j}\left(r, C^{\mathcal{P}}\right), c^{j}\right)=P_{i}\left(P_{j}\left(r, C^{\mathcal{P}}\right), c^{j}\right)=P_{i}^{d}(r, c, \mathcal{P})
$$

We can extend this proof to the family of decentralized rationing problems with rational claims $(r, c, \mathcal{P}) \in \mathcal{D}_{\mathbb{Q}}^{N}$ by simply splitting the agents' claims as follows. Notice that for all $i \in N, c_{i}=\frac{a_{i}}{b_{i}}$, where $a_{i} \in \mathbb{N}_{+}$and $b_{i} \in \mathbb{N}_{++}$. Then, if we denote by $l m$ the least common multiple of $\left(b_{i}\right)_{i \in N}$, we have that $c_{i}=\frac{a_{i} \cdot l m / b_{i}}{l m}$, for all $i \in N$, and, thus, $a_{i} \cdot l m / b_{i} \in \mathbb{N}_{+}$, for all $i \in N$. To complete the proof just split the agents' claims into several equal claims of value $\frac{1}{l m}$.

\section{Decentralized consistent standard rationing rules}

In this section we characterize those standard rationing solutions $F$ that assign the same allocation regardless of whether the resource is distributed directly or indirectly. If this occurs, we say that the standard rationing rule $F$ is a decentralized consistent rule.

Definition 4 A standard rationing rule $F$ satisfies decentralized consistency if for any arbitrary partition $\mathcal{P}=\left\{N_{j}\right\}_{j \in G}$ of $N$ it holds that

$$
F(r, c)=\mathcal{F}(r, c, \mathcal{P})
$$

where $\mathcal{F}$ is the decentralized rationing rule relative to $F$.

To obtain the characterization result, we need to introduce the concept of non manipulability of rules (de Frutos, 1999). A rule is non manipulable if no agent has an incentive to split her claim in several claims and no subset of agents has an incentive to merge their claims in a single claim.

Definition 5 A standard rationing rule $F$ is a non manipulable rule if for all $T, N \in \mathcal{N}$ with $T \subset N, T \neq \varnothing$, all $(r, c) \in \mathcal{R}^{N}$ and all $\left(r, c^{\prime}\right) \in \mathcal{R}^{T}$ such that $c_{i}^{\prime}=c_{i}+\sum_{k \in N \backslash T} c_{k}$, for some $i \in T$, and $c_{k}^{\prime}=c_{k}$, for all $k \in T \backslash\{i\}$, it holds that

$$
F_{i}\left(r, c^{\prime}\right)=F_{i}(r, c)+\sum_{k \in N \backslash T} F_{k}(r, c) .
$$


In the Introduction we have mentioned that decentralized rationing problems are a particular case of MIA situations. For MIA situations, Moreno-Ternero (2009) proves that the proportional rule is the only anonymous ${ }^{4}$ rule that assigns the same allocation directly or via a two-stage procedure. We next show that anonymity requirement can be dropped, in our context, from his characterization result.

Theorem 2 A standard rationing rule $F$ is a decentralized consistent rule if and only if it is the proportional rule.

Proof. First, we prove that if $F$ is a decentralized consistent rule, then $F$ is the proportional rule. Let $(r, c) \in \mathcal{R}^{N}$ and $T, N \in \mathcal{N}$ be two arbitrary finite sets of agents such that $T \subset N=$ $\{1,2, \ldots, n\}$ with $T \neq \emptyset$. Suppose w.l.o.g. that $T=\{1,2, \ldots, t\}$ and, thus, $N \backslash T=\{t+1, t+$ $2, \ldots, n\}$. Take an arbitrary agent $i \in T$ and consider the partition $\mathcal{P}=\{\{1\},\{2\}, \ldots,\{i-$ $1\},\{i\} \cup N \backslash T,\{i+1\}, \ldots,\{t\}\}$ of $N$; henceforth, we name $N_{i}=\{i\} \cup N \backslash T$. Notice that the components of the vector $C^{\mathcal{P}}$ are $C^{k}=c_{k}$, for all $k \in T \backslash\{i\}$ and $C^{i}=c_{i}+\sum_{k \in N \backslash T} c_{k}$. Hence,

$$
\begin{aligned}
F\left(r, C^{\mathcal{P}}\right) & =F\left(r,\left(C^{1}, C^{2}, \ldots, C^{i-1}, C^{i}, C^{i+1}, \ldots, C^{t}\right)\right) \\
& =F\left(r,\left(c_{1}, c_{2}, \ldots, c_{i-1}, c_{i}+\sum_{k \in N \backslash T} c_{k}, c_{i+1}, \ldots, c_{t}\right)\right) .
\end{aligned}
$$

On the other hand, since $F$ is a decentralized consistent rule (see Definition 4 ) and by efficiency of $F$, we obtain that

$$
\begin{aligned}
F_{i}(r, c)+\sum_{k \in N \backslash T} F_{k}(r, c) & =\mathcal{F}_{i}(r, c, \mathcal{P})+\sum_{k \in N \backslash T} \mathcal{F}_{k}(r, c, \mathcal{P})=\sum_{k \in\{i\} \cup N \backslash T} F_{k}\left(F_{i}\left(r, C^{\mathcal{P}}\right), c^{i}\right) \\
& =\sum_{k \in\{i\} \cup N \backslash T} F_{k}\left(F_{i}\left(r, C^{\mathcal{P}}\right),\left(c_{i}, c_{t+1}, c_{t+2}, \ldots, c_{n}\right)\right)=F_{i}\left(r, C^{\mathcal{P}}\right) .
\end{aligned}
$$

Thus, we conclude that $F$ is non manipulable. Since the unique non-manipulable rule is the proportional rule $P$ (see de Frutos, 1999), the proof of the "only if" part is done.

To prove the converse, we must show that $P$ is decentralized consistent, i.e. $P(r, c)=$ $P^{d}(r, c, \mathcal{P})$. By definition, we have that, for all $j \in G$ and all $i \in N_{j}$,

$$
P_{i}^{d}(r, c, \mathcal{P})=P_{i}\left(P_{j}\left(r, C^{\mathcal{P}}\right), c^{j}\right) .
$$

Then, we obtain that, for all $j \in G$ and all $i \in N_{j}$,

$$
P_{i}\left(P_{j}\left(r, C^{\mathcal{P}}\right), c^{j}\right)=P_{i}\left(r \cdot \frac{C^{j}}{\sum_{j \in G} C^{j}}, c^{j}\right)=r \cdot \frac{c_{i}}{\sum_{j \in G} C^{j}}=r \cdot \frac{c_{i}}{\sum_{i \in N} c_{i}}=P_{i}(r, c) .
$$

Therefore, by (8) and (9), we conclude that $P(r, c)=P^{d}(r, c, \mathcal{P})$, and thus, the "if" part of the proof is done.

Since the proportional rule is the unique decentralized consistent and non-manipulable rule, the next corollary follows.

Corollary 1 Decentralized consistency and non-manipulability are equivalent properties.

\footnotetext{
${ }^{4} \mathrm{~A}$ rule $F$ is anonymous if the allocation proposed by the rule does not depend on the name or label of players.
} 


\section{Acknowledgements}

The authors acknowledge support from research grant ECO2017-86481-P (Spanish Ministry of Science and Innovation, AEI and FEDER, UE) and 2017SGR778 (Government of Catalonia).

\section{References}

[1] Bergantiños, G., Lorenzo, L., \& Lorenzo-Freire, S., 2010. A characterization of the proportional rule in multi-issue allocation situations. Oper. Res. Lett. 38, 17-19.

[2] Bergantiños, G., Lorenzo, L., \& Lorenzo-Freire, S., 2011. New characterizations of the constrained equal awards rule in multi-issue allocation problems. Math. Methods Oper. Res. 74, $311-325$.

[3] Casas-Méndez, B., García-Jurado, I., van den Nouweland, A., \& Vázquez-Brage, M., 2003. An Extension of the $\tau$-Value to Games with Coalition Structures. European J. Oper. Res. 148, 494-513.

[4] de Frutos, M.A., 1999. Coalitional manipulation in a bankruptcy problem. Rev. Econ. Des. 4, $255-272$.

[5] Lorenzo-Freire, S., Casas-Méndez, B., \& Hendrickx, R., 2010. The two-stage constrained equal awards and losses rules for multi-issue allocation situations. TOP 18, 465-480.

[6] Moreno-Ternero, J., 2009. The proportional rule for multi-issue allocation bankruptcy problems. Econ. Bull. 29, 474-481.

[7] Thomson, W., 2015. Axiomatic and game-theoretic analysis of bankruptcy and taxation problems: An update. Math. Social Sci. 74, 41-59. 